\title{
BMJ Open Multimorbidity patterns in chronic older patients, potentially inappropriate prescribing and adverse drug reactions: protocol of the multicentre prospective cohort study MoPIM
}

\author{
Marisa Baré (D) ,1,2 Susana Herranz, ${ }^{3}$ Rosa Jordana, ${ }^{4}$ Maria Queralt Gorgas, ${ }^{5}$ \\ Sara Ortonobes, ${ }^{5}$ Daniel Sevilla, ${ }^{6}$ Elisabet De Jaime, ${ }^{7}$ Olatz Ibarra, ${ }^{8}$ \\ Candelaria Martín, ${ }^{9}$ on behalf of the MoPIM study group
}

To cite: Baré M, Herranz S, Jordana $\mathrm{R}$, et al. Multimorbidity patterns in chronic older patients, potentially inappropriate prescribing and adverse drug reactions: protocol of the multicentre prospective cohort study MoPIM. BMJ Open 2020;10:e033322. doi:10.1136/ bmjopen-2019-033322

- Prepublication history for this paper is available online. To view these files, please visit the journal online (http://dx.doi. org/10.1136/bmjopen-2019033322).

Received 31 July 2019 Revised 22 November 2019 Accepted 30 December 2019

Check for updates

(C) Author(s) (or their employer(s)) 2020. Re-use permitted under CC BY-NC. No commercial re-use. See rights and permissions. Published by BMJ.

For numbered affiliations see end of article.

Correspondence to

Dr Marisa Baré;

mbare@tauli.cat

\section{ABSTRACT}

Introduction Multimorbidity is a major challenge for current healthcare systems and professionals. From the different approaches that have been proposed to analyse this issue, the hypothesis of the existence of association patterns of different chronic conditions is gaining visibility. In addition, multimorbidity can be associated to polypharmacy, which can lead to a higher risk of potentially inappropriate prescribing (PIP) and consequently to adverse drug reactions (ADRs). The general objective of this novel study is to identify the association between PIP, multimorbidity patterns, polypharmacy and the presence of ADRs in older patients admitted for exacerbation of chronic diseases.

Methods and analysis The MoPIM (morbidity, potentially inappropriate medication) study is a multicentre prospective cohort study of an estimated sample of 800 older ( $\geq 65$ years) patients admitted to five general hospitals in Spain due to an exacerbation of a chronic disease. Patients referred to home hospitalisation, admitted due to an acute process or with a fatal outcome expected at the time of admission are excluded. Sociodemographic data, chronic morbidities and geriatric syndromes, number of chronic prescribed medications, PIP at admission to hospital and on discharge, according to the newest screening tool of older screening tool of older person's potentially inappropriate prescriptions/ screening tool to alert doctors to right treatment criteria, and ADRs during hospitalisation are being collected. Multimorbidity patterns will be identified using cluster analyses techniques, and the frequency of polypharmacy, PIP and ADRs will be calculated. Finally, the possible relationship between those indicators will be identified through bivariate and multivariate analyses.

Ethics and dissemination The project has been approved by the clinical research ethics committees of each centre: Comité Ético de investigación Clínica del Parc Taulí, Comitè Ètic d'Investigació Clínica Osona per a la Recerca i Educació Sanitàries (FORES), Comité de Ètica de la Investigación con Medicamentos (CEIm)-Parc de Salut MAR, Comité Ético de Investigación Clínica de Euskadi, Comité de Ética de Investigación del Hospital
Strengths and limitations of this study

- To our knowledge, this is the first published study that includes objectives related to chronic multimorbidity, appropriateness of medication and adverse drug reactions during hospitalisation at the same time, in older patients.

- Data of multimorbidity include also identification of chronic exacerbated diseases that cause hospitalisation, geriatric syndromes and functional status.

- The multicentre cohort design as well as the innovative analytical approach of multimorbidity patterns can lead to novel results in this field.

- Adverse drug reactions rate could be subject to an infra-estimation if active efforts to identify them are not complete.

Universitario de Canarias. The results will be actively and mainly disseminated through publication in peer-reviewed journals and communications in scientific conferences. Trial registration number NCT02830425.

\section{INTRODUCTION}

Multimorbidity and possible patterns

In recent years, the healthcare landscape has changed dramatically, with the considerable and gradual increase of older patients with multiple chronic health conditions. ${ }^{1}$ Multimorbidity has become an important challenge for the health system due to, on the one hand, the population ageing (which increases the percentage of people with multimorbidity) and, on the other hand, the difficulty of clinical management of patients with multimorbidity. ${ }^{12}$ Far from treating diseases or isolated processes, in patients with several chronic pathologies, and especially at times of decompensation, the complexity inherent to this type of patients becomes more evident. 
In addition, in older patients, a process of exacerbation or decompensation of chronic problems may coincide with the existence of geriatric syndromes such as acute confusional syndrome or frailty. In these cases, therapeutic decisions may require great knowledge and skills for sharing decision making to achieve an adequate balance between risks and benefits for the patient. ${ }^{3}$ However, clinical practice guidelines do not usually consider or resolve the uncertainties related to these situations. In fact, randomised controlled trials often exclude patients with concomitant pathologies. ${ }^{4}$

Currently, basic knowledge about the interrelationship of diseases is very limited, partly because of the existing confidence in a scientific method that maximises internal validity but excludes patients with comorbidity in both observational studies and clinical trials. Attempts to study multimorbidity clash with the absence of an internationally agreed definition and quantification. ${ }^{5}$ A systematic review of Violan et $a t^{6}$ identified important variations in both the definition of multimorbidity and its prevalence in different contexts and ages. Despite these variations, multimorbidity is much more frequent in older patients. ${ }^{78}$ Among the different definitions, the one that advocates for the existence of association patterns of some of these chronic diseases, either because they coincide, or because they share some physiopathological mechanisms, is recently gaining strength. ${ }^{910}$

\section{Polypharmacy, PIP and ADRs}

Polypharmacy is a remarkable feature of patients with multiple chronic pathologies. For this same reason, the prevalence of polypharmacy is usually high in the older population. ${ }^{11}$ Although there is no consensus on the minimum number of prescribed drugs to consider a patient as polymedicated, the chronic daily consumption of five or more drugs is a commonly accepted figure. ${ }^{12}$ Polypharmacy, in turn, is associated with a higher probability of adverse drug reactions (ADRs). In older people, this probability is even higher as a result of physiological changes associated with ageing, changes in the pharmacokinetic and pharmacodynamic behaviour of drugs, functional problems and social aspects. It has been estimated that ADRs are responsible for up to $30 \%$ of hospital admissions in older patients. ${ }^{13}$ Potentially inappropriate prescribing (PIP) of drugs and poor monitoring of prescribed treatments are predisposing factors for the appearance of ADRs. ${ }^{13}$ Fernandez et al $l^{14}$ observed that $69 \%$ of the ADRs detected in hospitalised polymedicated older patients were due to treatments that were identified as inappropriate. In 2011, Hamilton and colleagues also identified an association between overtreatment and ADRs in patients. ${ }^{15}$

\section{The STOPP-START criteria}

There are several tools to evaluate potentially inadequate prescribing. ${ }^{16-18}$ Among all of them, the explicit criteria STOPP-START (screening tool of older person's potentially inappropriate prescriptions/screening tool to alert doctors to right treatment) are the most used and validated in European older people. In fact, they were developed under the aegis of the European Union Geriatric Medicine Society. ${ }^{19}$ STOPP-START criteria include drug interactions and therapeutic duplicity. ${ }^{19}$ The 2008 version consisted of a list of 84 medication indications, developed using a Delphi method applied to experts from different disciplines, who carried out a literature review. The criteria are directed to prevalent diseases in older patients and are ordered by physiological systems. These criteria are easy to relate to active diagnoses and to the patients' medication lists that appear in their electronic health records. The systematic review published by Hill-Taylor et al in $2013^{16}$ concluded that these criteria were more sensitive than those of Beers to detect PIP. In 2015, O'Mahony et $a t^{20}$ published an updated version that includes 114 criteria, which was subsequently translated into Spanish. ${ }^{21}$

It should be noted that although the criteria for evaluating PIP can be helpful in making decisions, the clinical and social context of the patient has to be always taken into account. Hence there is need to share and assess the findings or PIP detected with the prescribing professional. Fortunately, it is increasingly common to use these or other criteria to identify a possible treatment inadequacy and assess a possible deprescription or prescription. However, its systematic use could be far from being real.

In recent years, many studies have been published using the STOPP-START criteria to assess the adequacy of medications in the community, socio-health centres and/ or nursing homes, and the hospital setting. ${ }^{14} 1822-28$ At the time of initiating this study, no published analyses were available with the objective to describe the relationships between multimorbidity, PIP and ADRs.

\section{Multimorbidity, polypharmacy, PIP and ADRs}

Given the importance of this phenomenon, it is necessary to deepen our knowledge to offer the best clinical care and optimise health outcomes.

Taking into account all the previous considerations, the hypotheses of the study were, first, that the morbidity of older patients admitted to hospital due to exacerbation of their chronic pathology may show certain patterns or profiles of association. Second, that geriatric syndromes may be relevant in these patterns, and that these patterns may be associated with greater polypharmacy and PIP. And third, that potentially inappropriate medications, or some of them, may, in turn, be associated with a greater presence of ADRs, already detected at the time of hospital admission or developed during the same hospitalisation, compared with those patients with no medication inappropriateness.

\section{Objectives}

The general objective of the study is to identify the association between multimorbidity, polypharmacy, PIP and the presence of ADRs in older patients admitted for 
exacerbation of chronic diseases. The specific objectives are to:

1. Identify and describe the PIP of chronic medication according to STOPP-START criteria at the time of admission and on discharge.

2. Estimate the prevalence of multimorbidity and, together with geriatric syndromes and risk factors, identify possible morbidity association patterns in older patients admitted as a result of an exacerbation of their chronic disease.

3 . Describe the possible polypharmacy ( $\geq 5$ chronic medications) of those patients.

4. Assess the difference in PIP on admission and at discharge.

5. Evaluate if any of the possible multimorbidity patterns are associated with greater PIP or with specific types of PIP.

6. Analyse the possible association between the amount or the types of PIP and the number or types of ADRs.

\section{METHODS AND ANALYSIS}

\section{Design and setting}

A multicentre prospective cohort study has been designed, including patients admitted to the internal medicine or geriatric services of five general hospitals in three different regions of Spain: Parc Taulí Hospital Universitari, Consorci Hospitalari de Vic, Hospital del Mar, Hospital de Galdakao and Complejo Hospitalario Universitario de Canarias. All of them have an internal medicine and/or geriatric service and are part of, or collaborate with, the Spanish Health Services Research on Chronic Patients Network (REDISSEC). In addition, a process of medication review is established in all of them, using criteria for older patients.

\section{Inclusion criteria}

Patients older than 64 years admitted to the internal medicine or geriatrics service as a result of the exacerbation or decompensation of their chronic pathology are included. A stay of $>48$ hours in hospital is considered an admission.

No written informed consent was deemed necessary for this study.

\section{Exclusion criteria}

Patients referred to home hospitalisation (patient at home but the clinical team provides punctual attention by telephone or home visits), those admitted due to an acute process not related to the chronic diseases (for example, an infection) according to clinical judgement of the corresponding physician or those with a fatal outcome expected at the time of admission are not included.

In the case that various patients met the inclusion criteria during the same day, and so that the clinical team could take the tasks of registering all variables of the study together with their care tasks, they were sorted alphabetically and selected the first of all of them to be included in this study.
Only patients attended by the physician participating in the study are included.

During recruitment, which lasted from September 2016 until the end of 2018, each patient was included only once.

\section{Data acquisition}

Each patient is monitored until hospital discharge (or death during hospitalisation), with data collection during the first days of admission to hospital ward and at the time of discharge.

As part of the usual patient care routine for older patients, the pharmacist of the clinical committee reviews the prescribed chronic medication at the time of admission. STOPP/START (SS) criteria are checked for each patient. These consist of 80 STOPP criteria (which detect medication that would not meet criteria for indication to a patient or a specific clinical situation or medications prescribed included drug-drug and drug-disease interactions) and 34 START criteria (detect medication that would be recommended to incorporate, including some vaccines).$^{20}$ For each patient, possible PIP are recorded at the time of admission and, following the usual practice, the clinical committee evaluates the PIP together with the possible modification of the medication according to the SS criteria. For the purposes of the study, the criteria are re-applied and recorded by the pharmacist to the prescribed medication on discharge, in this case without evaluation by the clinical committee.

In addition, ADRs notified to the pharmacy department by the clinical team or registered in the clinical course are identified by the pharmacist, and the consequences derived from them are classified as well as if they have been resolved at the time of discharge. According to the WHO and the European Medicine Agencies, an ADR is any undesirable event that has happened to the patient while using a medication and there is a suspicion that it is caused by the medication, including: (a) any noxious, unintended and undesired effect of a drug after doses used in humans for prophylaxis, diagnosis or therapy and (b) noxious and unintended effects resulting not only from the authorised use of a medicinal product at normal doses but also from medication errors and uses outside the terms of the marketing authorisation, including the misuse and abuse of the medicinal product. ${ }^{29}{ }^{30}$ As a routine standard practice, a physician, a nurse, a clinical assistant or the pharmacist can notify any ADRs to the pharmacy department or to the electronic notification system for adverse reactions to medication, held by the Spanish Ministry of Health.

\section{Variables}

The different types of information obtained according to the moment of monitoring are shown in figure 1. All of them are described below:

a. Sociodemographic: patient's code, centre, date of birth, sex, type of cohabitation (alone, with relatives or other people, in a nursing home). 


\section{Data registered for each patient}

\begin{tabular}{|l|l|l|}
\hline At arrival to hospital ward & During hospitalization & At discharge \\
- Age, sex, living conditions & - New ADR identified & - New geriatric syndromes \\
- Date of arrival to emergency room and & - Treatment involved & - Number of prescribed \\
date of arrival to in-hospital ward & - Date of onset & - PIP newly detected \\
- Contact with health system in previous 3 & - Type & - Date of discharge \\
months for chronic disease exacerbation & - Consequence & - Destination \\
- Barthel Index before in-hospitalisation & - Resolved or not & - Reason of death: \\
- Chronic morbidity & - Clinical decision for each PIP & - Chronic disease \\
- Chronic morbidity exacerbated & - To maintain treatment & treatment \\
- Geriatric syndromes & - To change treatment & - Other \\
- Number of prescribed chronic medications & - To stop treatment & \\
- Other non pharmacologic treatments & - To start treatment & \\
- Allergies to medication & & \\
- PIP of chronic medication & & \\
- ADR identified at admission & & \\
- Treatment involved & & \\
- Type & & \\
& &
\end{tabular}

Figure 1 Data registered for each patient included in the morbidity, potentially inappropriate medication study. $A D R$, adverse drug reactions; PIP, potentially inappropriate prescribing.

b. Clinical: date of arrival at the emergency room, date of admission, date of discharge, destination at discharge (home, transfer to another hospital, transfer to another service of the hospital itself, transfer to a nursing home), cause of the death (chronic illness, complication or others), functional status just before entering the hospital (Barthel index), existence of hospitalisation and/or visits to healthcare services in the three previous months due to exacerbation of any chronic pathology, existence of pharmacological allergies and active principles involved.

Chronic active diseases of the patient are recorded. For this purpose, the physicians of the project have defined, on a consensual basis, a limited list of 64 chronic problems or conditions, coming from the 114 groups defined by Salisbury and colleagues, ${ }^{8}$ and including the 19 categories of the Charlson Index. ${ }^{31}$ In order to define the list of conditions to be recorded, the physicians that participated in the project considered the subjective estimated frequency of the condition in older hospitalised patients in their departments. Following the same criteria as Salisbury, a condition is considered to be chronic when it lasts for at least 6 months, including past conditions that require ongoing disease or risk management, important conditions with a significant risk of recurrence or past conditions that have continuing implications for patient management. ${ }^{8}$

For each of the chronic condition (see table 1), it is also recorded if they have required attention or clinical management throughout the hospitalisation, and the attending physician of the clinical team assigns a (subjective) correlative score $(1,2,3 \ldots)$ to each one, according to the importance or weight during the attention process. Thus, chronic problems that have not had any significance during hospitalisation do not represent any score. Drug-related conditions of this list refer to poor management of medication for a chronic disease that have clinical
Table 1 Chronic conditions and geriatric syndromes recorded

\begin{tabular}{ll}
\hline Chronic conditions & $\begin{array}{l}\text { Geriatric syndromes } \\
\text { and risk factors }\end{array}$ \\
\hline Charlson Index & $\begin{array}{l}\text { Acute confusional } \\
\text { syndrome/delirium }\end{array}$ \\
\hline 1. AIDS/HIV & Chronic pain \\
\hline 2. Any malignancy (excluding skin) & $\begin{array}{l}\text { Cognitive/intellectual } \\
\text { impairment }\end{array}$ \\
\hline 3. Cerebrovascular disease & Constipation \\
\hline 4. Chronic pulmonary disease & Depression or Anxiety \\
\hline 5. Congestive heart failure & Dysphagia \\
\hline 6. Dementia & Frailty \\
\hline 7. Diabetes with complication & Immobility \\
\hline 8. Diabetes without complication & $\begin{array}{l}\text { Incontinence (Urinary/ } \\
\text { faecal) }\end{array}$ \\
\hline 9. Hemiplegia & Instability/falls \\
\hline 10.Leukaemia & Malnutrition \\
\hline 11.Lymphoma & Polypharmacy \\
\hline 12.Metastatic solid tumour & Pressure ulcers \\
\hline 13.Mild liver disease & Sensorial deficit \\
\hline 14.Moderate or severe liver disease & $\begin{array}{l}\text { Sleep disorders/ } \\
\text { Insomnia }\end{array}$ \\
\hline 15.Moderate or severe renal diseas
\end{tabular}

16.Myocardial infarction

17.Peptic ulcer disease

18.Peripheral vascular disease

19.Rheumatologic disease

\section{Other conditions}

20.Amputation

21.Anaemia

22.Asthma

23.Cardiac arrhythmia

24. Cataract

25.Chronic hepatitis (B or C)

26. Chronic pancreatic disease

27.Degenerative arthropathy

28.Dermatitis or eczema

29. Diverticular disease of the colon

30.Drug-related conditions

31.Dyslipidaemia

32.Fibromyalgia

33.Gallstones (previous hepatic colic)

34.Chronic gastritis or gastro-oesophageal reflux

35. Glaucoma

36.Gout

37. Haemorrhoids

38. Haematologic disorders (myelodysplastic syndrome, gammapathy, polycythaemia) 
Table 1 Continued

\section{Chronic conditions}

Geriatric syndromes and risk factors

40. Inflammatory osteoarticular disease

41.Irritable bowel syndrome

42.Ischaemic heart disease without infarction

43.Migraine

44.Neurologic disorder of the central nervous system

45.Non-congestive heart failure

46.Non-ischaemic heart disease (miocardiopatie, valvulopatie)

47.Non-schizophrenic mental disorders (excluding depression and anxiety)

48.Obesity

49. Osteoporosis

50.Other neurological pathologies (essential tremor)

51.Other vascular diseases (ischaemia, aneurism)

52.Parkinson's disease

53.Peripheral neuropathy or neuritis

54.Post-traumatic stress disorder

55.Previous fractures (not hip)

56.Previous hip fracture

57.Prostatic benign hypertrophy

58.Schizophrenia

59.Sleep apnoea

60.Chronic thyroid disease

61.Tuberculosis

62.Urinary tract stones (nephritic colic)

63.Varicose veins of lower extremities

64.Vertigo

implications in that hospitalisation (as, for example, any drug intolerance or an excess drug poisoning).

Specific geriatric syndromes and risk factors (acute confusional syndrome/delirium, chronic pain, cognitive/intellectual impairment, constipation, depression or anxiety, dysphagia, frailty, immobility, incontinence (urinary/faecal), instability/falls, malnutrition, polypharmacy, pressure ulcers, sensorial deficit, sleep disorders/ insomnia) are also recorded, as usual. Two of the departments systematically apply a recently developed scale for frailty, ${ }^{32}$ while the others consider clinical judgement (although based on the same variables).

c. Pharmacological: Number of chronic medications of the electronic prescribing at the time of admission and discharge, SS criteria detected on admission, active principle involved, clinical decisions to modify the prescription associated with the PIP detected, SS criteria detected on discharge and the active principle involved in the PIP detected. Medication is only considered chronic if it has been prescribed, at least, 3 months ago. Active principles were only considered separately, regardless of the combinations. Creams, ointments and healing material were not considered.

d. ADRs identified both at the time of admission and during the course of admission: drug involved, type of ADR according to the Wills and Brown classification ${ }^{33}$ (predictable, unpredictable, continuous or prolonged, with carcinogenic effect, rebound effect), start date if it appeared along stay in the hospital, consequences in terms of health (causes death, threatens life, lengthens the time of hospitalisation, other important consequences under medical criteria) and if they are resolved during admission or at discharge.

\section{Pilot study}

At the beginning of the recruitment, the first 10 admissions per centre were selected to validate the data collection instruments and identify the problems that could arise during this stage. Then, relevant changes were made in the protocol and in the questionnaires.

\section{Sampling and analysis}

An estimated consecutive sample of 800 patients meeting the inclusion criteria are being selected, proportionally distributed to the volume of income of the medicine and/or geriatric services of each centre.

The sample size calculation was based on the indicator 'Prevalence of patients with PIP'. Taking into consideration the variability of the prevalence for older patients in a published review, ${ }^{16}$ that ranged between $21 \%$ and $79 \%$ of cases fulfilling any STOPP/START criteria, estimation was based on a prevalence of PIP of $50 \%$ (which would provide the highest number of patients to include), an alpha risk of $5 \%$ and a power of $80 \%$. Then, a minimum of 400 patients would be required for estimation of the prevalence of PIP in the whole sample. In order to increase the statistical power of the bivariate and multivariate analyses, and specifically the proposed cluster analysis, for which there is no sample calculation formula, and taking also into account the feasibility and possible difficulties of each centre and the funding of the project, the initial sample size was increased by $100 \%$.

According to the objectives of the study, the following steps of data analysis will be performed:

\section{The main descriptive indicators will be:}

- Number and percentage of patients with PIP, both on admission and at discharge.

- Number and percentage of the STOPP-START criteria identified on admission and at discharge.

- Number and percentage of polymedicated patients ( $\geq 5$ chronic active principles).

- Number and percentage distribution of chronic pathologies.

- Number and percentage of geriatric syndromes and risk factors.

- Number and percentage of the chronic pathologies that have motivated admission due to exacerbation. 
- Number and percentage of patients with any ADR detected on or during admission.

b) The possible multimorbidity patterns will be identified using a cluster analysis algorithm, similar to that used by Marengoni $e t a l .^{34}$ With that intention, diseases or syndromes with a very low prevalence will be excluded to avoid statistical noise and therefore spurious findings in the cluster solutions. To characterise the multimorbidity patterns corresponding to each cluster of individuals, the frequency of chronic diseases or syndromes in each cluster will be calculated. Observed/expected ratios will be calculated by dividing the prevalence of a given disease within a cluster by its prevalence in the overall population. The exclusivity of different diseases, defined as the fraction of participants with the disease included in the cluster over the total number of participants with the disease, will be also calculated.

c) To evaluate the relationship between multimorbidity and PIP, and to analyse the possible association between PIP and ADRs, a bivariate analysis will be carried out, where the variables will be treated quantitatively and/or categorically (number of morbidities, number of PIP or ADRs, patterns of morbidity, existence of PIP or ADRs, types of PIP or ADRs). Parametric or non-parametric tests will be applied according to the normal distribution of the PIP or ADRs number. In addition, ORs and their CI will be estimated at $95 \%$ among multimorbidity patterns, existence of PIP as well as between PIP and ADRs detected at the time of admission.

d) Finally, multiple regression and multilevel analysis techniques (where the levels would be the patient and the hospital) will be applied to adjust for other possible factors (such as age, sex, Barthel index or previous visits) statistically significant in the bivariate analysis or those clinically relevant, where the dependent variable, PIP or ADRs, according to objective, may be dichotomous or quantitative. The adjusted $\beta$ coefficients and their $95 \% \mathrm{CI}$, and the exponentials of $\beta$ (OR) will be estimated according to whether it is a multiple linear regression or a generalised estimating equation model.

All analyses will be performed with $\mathrm{R}$ ( $\mathrm{R}$ Foundation for Statistical Computing, Vienna, V.3.6.0) and SPSS (IBM Corp, V.25.0). First results are expected to be available by the end of 2019.

\section{Patient and public involvement}

Since this is an observational study with variables and outcomes related to the healthcare process, this research is being developed without patient involvement. Patients are not invited to comment on the study design and are not consulted to develop patient relevant outcomes or interpret the results.

\section{ETHICS AND DISSEMINATION}

All the personal data will be anonymised, assigning a code to each patient, exclusive of the study, so that they no longer relate to identifiable people.
The dissemination plan includes publication in peerreviewed journals of several disciplines such as internal medicine, geriatrics or public health, as well as research communications in some scientific conferences and mass media. Other kind of dissemination activities to clinicians, managers and policy makers will be done in the proper format.

\section{Author affiliations}

${ }^{1}$ Department of Epidemiology and Cancer Screening, Consorci Corporació Sanitària Parc Taulí, Sabadell, Catalonia, Spain

${ }^{2}$ Department of Pediatrics, Obstetrics and Gynecology, Preventive Medicine and Public Health, Faculty of Medicine, Universitat Autònoma de Barcelona, Bellaterra, Catalonia, Spain

${ }^{3}$ Internal Medicine Department, Acute Care Geriatric Unit, Consorci Corporació Sanitària Parc Taulí, Sabadell, Catalonia, Spain

${ }^{4}$ Internal Medicine Department, Consorci Corporació Sanitària Parc Taulí, Sabadell, Catalonia, Spain

${ }^{5}$ Pharmacy Department, Consorci Corporació Sanitària Parc Taulí, Sabadell, Catalonia, Spain

${ }^{6}$ Pharmacy Department, Consorci Hospitalari de Vic, Vic, Catalonia, Spain ${ }^{7}$ Geriatrics Department, Consorci Parc de Salut MAR de Barcelona, Barcelona, Catalonia, Spain

${ }^{8}$ Pharmacy Department, Hospital Galdakao-Usansolo, Galdacano, País Vasco, Spain ${ }^{9}$ Internal Medicine Department, Hospital Universitario de Canarias, La Laguna, Canarias, Spain

\section{Twitter Marisa Baré @BareMarisa}

Acknowledgements The authors acknowledge the dedication and support of the entire morbidity, potentially inappropriate medication research group.

Collaborators Morbidity, potentially inappropriate medication study group: Parc Taulí University Hospital: Marisa Baré (Clinical Epidemiology and Cancer Screening; REDISSEC), Susana Herranz (Acute Care Geriatric Unit), Rosa Jordana (Department of Internal Medicine), Maria Queralt Gorgas (Pharmacy Department, REDISSEC), Mònica Gómez and Sara Ortonobes (Pharmacy Department), Marina Lleal (Fundació Parc Taulí, REDISSEC); University Hospital of Vic: Pere Roura (Epidemiology Unit; REDISSEC), Daniel Sevilla, Núria Solà and Javier González (Pharmacy Department), Núria Molist, Mariona Espauella (Department of Geriatrics); Oscar Mascaró (Department of Internal Medicine); Hospital del Mar Medical Research InstituteIMIM: Elisabet de Jaime, Miguel Ángel Márquez, Marta Arellano, Carlos Clemente and Olga Sabartés (Department of Geriatrics), Olivia Ferrandez, Núria Carballo and Marta de Antonio (Pharmacy Department), Priscila Giraldo and Maria Sala (Department of Epidemiology and Evaluation, REDISSEC); Hospital de Galdakao: Rafael Estrada (Department of Internal Medicine), Maria Olatz Ibarra (Pharmacy Department); Complejo Hospitalario Universitario de Canarias: Candelaria Martin (Department of Internal Medicine), Gloria Julia Nazco (Pharmacy Department), Rubén Hernández (Department of Internal Medicine).

Contributors $\mathrm{MB}$, the principal investigator, conceived the study and wrote the protocol, in collaboration with SH, RJ, and MQG. SO, DS, EDJ, Ol and CM reviewed and made amendments to its preliminary version. They also contributed to the design of the final questionnaires and were responsible of the organisation of the fieldwork in each centre. All of the members of the study group participated in the inclusion of patients, as well as in the collection and quality assessment of clinical data for each patient.

Funding This work was supported by grants from Instituto de Salud Carlos IIIFEDER, [P115/00552] and by the Network for Research into Healthcare in Chronic Diseases, REDISSEC (RD16/0001/0002).

Competing interests None declared.

Patient consent for publication Not required.

Provenance and peer review Not commissioned; externally peer reviewed.

Open access This is an open access article distributed in accordance with the Creative Commons Attribution Non Commercial (CC BY-NC 4.0) license, which permits others to distribute, remix, adapt, build upon this work non-commercially, and license their derivative works on different terms, provided the original work is properly cited, appropriate credit is given, any changes made indicated, and the use is non-commercial. See: http://creativecommons.org/licenses/by-nc/4.0/. 
ORCID iD

Marisa Baré http://orcid.org/0000-0001-8085-2833

\section{REFERENCES}

1 Palladino R, Pennino F, Finbarr M, et al. Multimorbidity and health outcomes in older adults in ten European health systems, 2006-15. Health Aff 2019;38:613-23.

2 The Academy of Medical Sciences. Multimorbidity: a priority for global health research, 2015. Available: https://acmedsci.ac.uk/filedownload/82222577

3 Zulman DM, Asch SM, Martins SB, et al. Quality of care for patients with multiple chronic conditions: the role of comorbidity interrelatedness. J Gen Intern Med 2014;29:529-37.

4 Buffel du Vaure C, Dechartres A, Battin C, et al. Exclusion of patients with concomitant chronic conditions in ongoing randomised controlled trials targeting 10 common chronic conditions and registered at ClinicalTrials.gov: a systematic review of registration details. BMJ Open 2016;6:e012265.

5 Calderón-Larrañaga A, Vetrano DL, Ferrucci L, et al. Multimorbidity and functional impairment-bidirectional interplay, synergistic effects and common pathways. J Intern Med 2019;285:255-71.

6 Violan C, Foguet-Boreu Q, Flores-Mateo G, et al. Prevalence, determinants and patterns of multimorbidity in primary care: a systematic review of observational studies. PLoS One 2014;9:e102149.

7 Fortin M, Stewart M, Poitras M-E, et al. A systematic review of prevalence studies on multimorbidity: toward a more uniform methodology. Ann Fam Med 2012;10:142-51.

8 Salisbury C, Johnson L, Purdy S, et al. Epidemiology and impact of multimorbidity in primary care: a retrospective cohort study. Br J Gen Pract 2011;61:e12-21.

9 Poblador-Plou B, van den Akker M, Vos R, et al. Similar multimorbidity patterns in primary care patients from two European regions: results of a factor analysis. PLOS One 2014;9:e100375.

10 Prados-Torres A, Calderón-Larrañaga A, Hancco-Saavedra J, et al. Multimorbidity patterns: a systematic review. J Clin Epidemiol 2014;67:254-66.

11 Gutiérrez-Valencia M, Aldaz Herce P, Lacalle-Fabo E, et al. Prevalence of polypharmacy and associated factors in older adults in Spain: data from the National health survey 2017. Medicina Clínica 2019;153:141-50.

12 Masnoon N, Shakib S, Kalisch-Ellett L, et al. What is polypharmacy? A systematic review of definitions. BMC Geriatr 2017;17.

13 Cabré M, Elias L, Garcia M, et al. Avoidable hospitalizations due to adverse drug reactions in an acute geriatric unit. Analysis of 3,292 patients. Medicina Clínica 2018;150:209-14.

14 Fernández-Regueiro R, Fonseca-Aizpuru E, López-Colina G, et al. Prescripción inadecuada $Y$ efectos adversos a medicamentos en pacientes de edad avanzada. Rev Clin Esp 2011;221:400-6.

15 Hamilton H, Gallagher P, Ryan C, et al. Potentially inappropriate medications defined by STOPP criteria and the risk of adverse drug events in older hospitalized patients. Arch Intern Med 2011;171:1013-9.

16 Hill-Taylor B, Sketris I, Hayden J, et al. Application of the STOPP/START criteria: a systematic review of the prevalence of potentially inappropriate prescribing in older adults, and evidence of clinical, humanistic and economic impact. J Clin Pharm Ther 2013;38:360-72.
17 Patterson SM, Cadogan CA, Kerse N, et al. Interventions to improve the appropriate use of polypharmacy for older people. Cochrane Database Syst Rev 2014:CD008165.

18 Motter FR, Fritzen JS, Hilmer SN, et al. Potentially inappropriate medication in the elderly: a systematic review of validated explicit criteria. Eur J Clin Pharmacol 2018;74:679-700.

19 Gallagher P, Ryan C, Byrne S, et al. STOPP (screening tool of older Person's prescriptions) and start (screening tool to alert doctors to right treatment). consensus validation. Int J Clin Pharmacol Ther 2008;46:72-83.

20 O'Mahony D, O'Sullivan D, Byrne S, et al. STOPP/START criteria for potentially inappropriate prescribing in older people: version 2. Age Ageing 2014;44:213-8.

21 Delgado Silveira E, Montero Errasquín B, Muñoz García M, et al. Mejorando La prescripción de medicamentos en Las personas mayores: Una nueva edición de Los criterios STOPP-START. Revista Española de Geriatría y Gerontología 2015;50:89-96.

22 García-Gollarte F, Baleriola-Júlvez J, Ferrero-López I, et al. Inappropriate drug prescription at nursing home admission. J Am Med Dir Assoc 2012;13:83.e9-83.e15.

23 Sevilla-Sánchez D, Molist-Brunet N, Amblàs-Novellas J, et al. Potentially inappropriate medication at hospital admission in patients with palliative care needs. Int J Clin Pharm 2017;39:1018-30.

24 San-José A, Agustí A, Vidal X, et al. Inappropriate prescribing to older patients admitted to hospital: a comparison of different tools of misprescribing and underprescribing. Eur J Intern Med 2014;25:710-6.

25 Salgueiro E, Elizarde BC, Elola Al, et al. [The most common STOPP/ START criteria in Spain. A review of the literature]. Rev Esp Geriatr Gerontol 2018:53:274-8.

26 Moriarty F, Bennett K, Cahir C, et al. Potentially inappropriate prescribing according to STOPP and START and adverse outcomes in community-dwelling older people: a prospective cohort study. $\mathrm{Br} \mathrm{J}$ Clin Pharmacol 2016;82:849-57.

27 Gibert P, Cabaret M, Moulis M, et al. Optimizing medication use in elderly people in primary care: impact of STOPP criteria on inappropriate prescriptions. Arch Gerontol Geriatr 2018;75:16-19.

28 Thomas RE, Thomas BC. A systematic review of studies of the STOPP/START 2015 and American geriatric Society beers 2015 criteria. Curr Aging Sci 2019;26:187-92.

29 European Medicines Agency and Heads of Medicines Agencies. Guideline on good pharmacovigilance practices (GVP) module $\mathrm{VI}$ - management and reporting of adverse reactions to medicinal products (Rev 1, 2014

30 Mascolo A, Scavone C, Sessa M, et al. Can causality assessment fulfill the new European definition of adverse drug reaction? A review of methods used in spontaneous reporting. Pharmacol Res 2017;123:122-9.

31 Charlson ME, Pompei P, Ales KL, et al. A new method of classifying prognostic comorbidity in longitudinal studies: development and validation. J Chronic Dis 1987;40:373-83.

32 Amblàs-Novellas J, Martori JC, Espaulella J, et al. Frail-VIG index: a concise frailty evaluation tool for rapid geriatric assessment. $B M C$ Geriatr 2018;18:29.

33 Wills S, Brown D. A proposed new means of classifying adverse reactions to medicines. Pharm J 1999;262:163-5.

34 Marengoni A, Roso-Llorach A, Vetrano DL, et al. Patterns of multimorbidity in a population-based cohort of older people: sociodemographic, lifestyle, clinical, and functional differences. $J$ Gerontol A Biol Sci Med Sci 2019;72. 